\title{
Integrated modelling approach for enhancing brain MRI with flexible pre-processing capability
}

\author{
Harish S. ${ }^{1}$, G. F. Ali Ahammed ${ }^{2}$ \\ ${ }^{1}$ VTU Belagavi, India \\ ${ }^{2}$ VTU PG-Center, Mysuru, India
}

\begin{tabular}{l} 
Article Info \\
\hline Article history: \\
Received Jun 19, 2018 \\
Revised Dec 21, 2018 \\
Accepted Mar 4, 2019
\end{tabular}

Keywords:

Brain

Enhancement

Magnetic Resonance Imaging

Pre-Processing

Resolution

\begin{abstract}
The assurance of an information quality of the input medical image is a critical step to offer highly precise and reliable diagnosis of clinical condition in human. The importance of such assurance becomes more while dealing with important organ like brain. Magnetic Resonance Imaging (MRI) is one of the most trusted mediums to investigate brain. Looking into the existing trends of investigating brain MRI, it was observed that researchers are more prone to investigate advanced problems e.g. segmentation, localization, classification, etc considering image dataset. There is less work carried out towards image preprocessing that potential affects the later stage of diagnosing. Therefore, this paper introduces a novel model of integrated image enhancement algorithm that is capable of solving different and discrete problems of performing image pre-processing for offering highly improved and enhanced brain MRI. The comparative outcomes exhibit the advantage of its simplistic implemetation strategy.
\end{abstract}

Copyright () 2019 Institute of Advanced Engineering and Science. All rights reserved.

\section{Corresponding Author:}

Harish S.,

Research Scholar, VTU Belagavi, India

Email: harishsrinivasaiah@gmail.com

\section{INTRODUCTION}

In the current scenario of radiological diagnosis, various medical imaging techniques have emerged as advantageous tool to serve a beneficial feature to medical practioners and experts in order to detect and cure abnormalities in human body in eaiser manner and with lower mental risk [1]. Images captured from various devices using different modes such as X-ray, CT scan, MRI and ECG. Among these imaging models MRI is considered to be very potential and trust full tool to visualize the complex and internal parts of human organs in detailed. The MRI stands for Magnetic Resonance Imaging that construct image using radio waves and magnetic field and it is largly adopted in era of neurological system for analysis of brain disorders such as tumors, cancer, lesions, etc. [2]. However, there are various challenges and issues associated with the visualization of brain MRI due to noises such as thermal noise, sample noise, incorporated noise, poor contrast, lack of boundaries and etc. Such phenomenon degrades the quality of image and makes difficult to analyze, recognize tumor in the brain. Thus, medical image processing techniques is considered as a solution to mitigate the problem of noise occurred in image inorder to provide suitable diagnosis of tumor in brain [3]. The image processing techniques includes several processes such as enhancement, denoising, segnmentation, localization, correction and etc. The denoising and segmentation imaging processing technique provides better analysis of brain tumor from an enhanced image. However, there is conflict between the image enhancement and denoising process because denoising is the process of mitigating noise from the image signal and enhancement is the process of improving and enhacing the quality of image by manipulating the digital image with some computational applications [4]. Furthermore, it has been noticed that there are various significant research work towards addressing segmentation problems as well as classification problems pertaining to brain MRI [5-8]. It can be seen that the existing research community is completely 
absorbed in evolving up new techniques of advance applications of medical image processing that usually includes segmentation, classification, and localization. However, essential techniques e.g. image preprocessing is quite found ignored and have not witnessed a notable progress. This is also another prime reason why even the advance processing technqiues are not found $100 \%$ successful. The reason is that image enhancement being the primary step is found highly ignored. Preprocessing of images is the very crucial step required to obtain accurate image visualization for proper diagnosis Medical images. The medical image consists of several artifacts and noises that create complexity in reading of medical images. There are various preprocessing techniques such as Adaptive Median Filter, Morphological Operations, Mean Filter or Average Filter, Histogram Equalization, Spatial Domain Enhancement Method, etc are used to enhance the medical image for better visibility [9-10]. There are several algorithms proposed in review of literature for achieving better readability of brain MRI images.

This paper presents a discussion of a novel and simple solution that targets to address the research gap associated with image preprocessing of brain MRI. The organization of the proposed manuscript is as following: - Section 1.1 discusses about the existing literatures where different techniques are discussed for detection schemes used in power transmission lines followed by discussion of research problems in Section 1.2 and proposed solution in 1.3. Section 2 discusses about algorithm implementation followed by discussion of result analysis in Section 3. Finally, the conclusive remarks are provided in Section 4.

This study discussed about various research attempts toward research-based techniques on brain MRI Images. The work carried out by Min and Kyu [11] have used fusion technique for Brains MRI image enhancement and clustering technique for tumor segmentation. Alex et al. [12] have applied machine learning approach with classicical autoencoder inorder to perform tumer segmentation task of brain MRI. Ortiz et al. [13] have constructed a featured framework that based on unsupervised techniques that contains both vector quantization and fuzzy system for brain MRI segmentation. Abbasi and Tajeri Pour [14] have considered joint approach of classification and clustering to detect the brain tumor from MRI images. Gupta and Porwal [15] have used Image enhancement Alogorithm inorder to enhance the visibility of Brain and Breart cancer medical Images. In the work of Menze et al. [16] have reported about the performance of Brain Tumor MRI Segmentation multimodel that conducted in MICCAI 2012 and in conferences 2013. Bhaskarrao et al. [17] have made their effort based on Berkeley wavelet transformation with SVM classifier in order to reduce the complexity occurs in medical image segmentation operation. Dhage et al. [18] have carried watershed image segmentation approach of brain MRI analysis for differentiating normal and abnorl mal barin tissue. The study of Faisal et al. [19] have utilized mathematical modeling for reducing noise with improved segmentation technique for detecting tumor from 2D barin MRI images.

In [20] Jui et al. have presented a new approach based on feature extraction for achieving better accuracy in medical image segmentation. Kaur and Rani [21] have introduced a histogram technique as effective approach for enhancing contrast and brightness of medical images. Megersa and Alemu [22] have designed an automated framework based on neural network composed of preprocessing, image fusion, classification task for automatic image segmentation and tumor detection form brain MRI. In [23] the Parveen and Singh have used combined technique that composed of SVM and data clustering technique inorder to enhance the MRI images for predicting brain tumor. Pereira et al. [24] have discussed about MRI as an effective way to analyze the brain tumer and the author have presented a model based on the Convolutional neural network in order to achieve a reliable segmentation of MRI. In work of [25] Rao et al. have considered a class of statistical modeling technique to classify and detect the region of brain tumor in the MRI.

In the same way Senthilkumaran and Thimmiaraja [26] have used Histogram equalization and Sharma and Meghrajani [27] have used mathematical morphological model to enhance the medical image visibility for efficiently analyzing barin tumor from MRI. The work of Sulaiman et al. [28] presented a new model based on the clustering algorithm, which objective is to remove the salt and pepper niose from the brain MRI. Xiao et al. [29] have introduced a novel method of lateral ventricular compression and deformation for tumor segmentation and detection. Zeljkovic et al. [30] have developed an intelligent framework composed of multiple tasks such as enhancement, segmentation, classification and tumor analysis from the Brain MRI. The proposed framework achieves good accuracy rate in automatic segmentation process compared to mannul segmentation process. The work is done by Hamiane and Saeed [31] shows the SVM categorization of MRI Brain images for computer helped studies. Jung et al. [32] have presented a method of altering the cluster optimal before cluster processing in the obtainable $\mathrm{K}$-means practice into a flexible cluster optimal through principal module study, and enhancing the possibility of data clustering. Lafta et al. [33] have demonstrated a method for diagnosing a set of diseases containing two kinds of cancer (lung as well as breast cancer), two datasets for heart attack and diabetes. The next section briefs of the problems extracted from the above approaches in analyzing brain MRI. 
The significant research problems are as follows:

1. Majority of the existing studies towards analyzing brain MRI are related to problems of segmentation and classification while very less work towards addressing enhancement issue.

2. Usage of image enhancement techniques are either very simple or very much complicated that doesn't offer much practical insight of the real-usage in the diagnosis process.

3. Usage of existing system towards image analysis is highly iterative and there are very less simplified techniques available for brain MRI images.

4. There is also less availability of joint mechanism to deal with multiple number of problems associated with image preprocess.

Therefore, the problem statement of the proposed study can be stated as "Developing a computational model that can jointly address multiple problems associated with image enhancement exclusively related to brain MRI is challenging". The next section outlines the proposed solution to address this problem.

The prime aim of the proposed study is to offer solutions towards some of the frequently occurring problems that act as impediment in analyzing medical images especially brain MRI. From the prior section, it has been seen that there are problems associated with homogenity of pixel information, noise inclusion, and complex implementation process that adversely affects the advance forms of analysis over the brain MRI image. Therefore, the proposed system offers three different set of solution in order to address this problem that is directly related to image enhancement. Figure 1 highlights the schematic diagram of proposed solution.
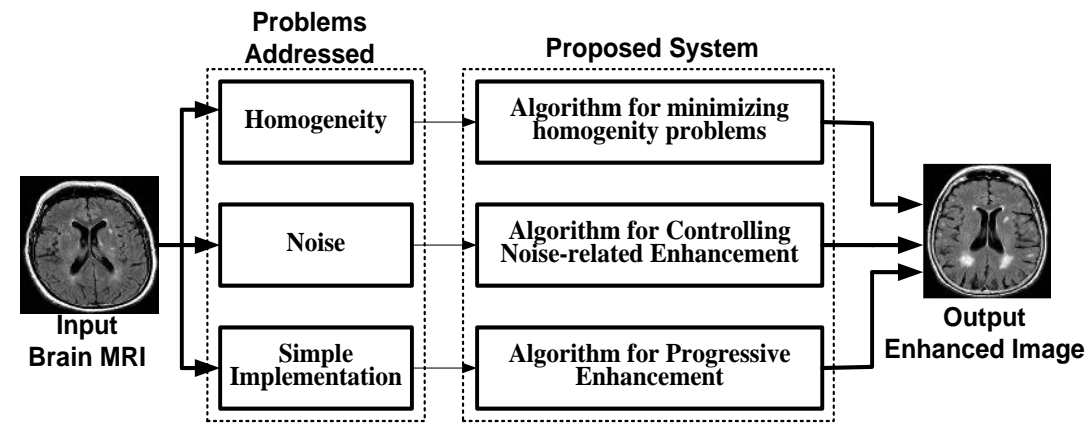

Figure 1. Schematic diagram of proposed solution

According to the proposed scheme, the prime ideology of the proposed implementation scenario is that different brain MRI images have different inclusion of requirements of preprocessing whereas only single algorithm is executed to perform image enhancement. This problem is solved by introducing three different schemes of implementation that emphasizes on addressing the homogenity problems, noise-relaed issues, as well as simple implementation issues. As brain MRI images are usually darker and the proportions of the dark pixels are comparatively more than MRI of other organs of human body, it is essential that there the image could be subjected to algorithms that is capable of performing pre-processing with higher degree of flexibility. By adopting the analytical research methodology, the proposed system is designed to offer three individual and discrete set of image enhancement operation in order to assist in diagnosing brain MRI image more closely. The advantages of the proposed system are as follows:

i) The proposed algorithm offers solution to multiple problems associated with image enhancement that are frequently encountered in existing research-based implementation,

ii) The proposed system is assessed using novel performance parameter that ensure proper assessment of contrast, brightness, sharpness, locality, execution performance all together.

iii) All the three sets of algorithms are meant to address different problems; however, they can also be interchangeably used if multiple such problems are present in the given input image,

iv) It saves time and offers more productivity to physician, who can perform closer study of given input of brain MRI image with wider ranges of selection of image enhancement operation. Apart from the above-mentioned advantages, the proposed system also offers optimization to simple filtering process which will infer a fact that it is feasible to perform non-recursive operation in order to obtain highly enhanced image. The next section illustrates about the algorithms being implemented. 


\section{ALGORITHM IMPLEMENTATION}

This section presents discussion of all the core algorithms that works in an integrated manner to offer a comprehensive enhancement of the brain MRI image. The ideology of this algorithm construction is that different brain MRI images will have different forms of problems which are required to be addressed in preprocessing stages itself. The proposed system discusses about three different possibilities of solutions to eleviate the image enhancement process using simple process of implementation.

\subsection{Solution towards homogeneity problems of brain MRI}

As seen in existing system, there are various forms of approaches for stretching the cumulative gray levels of the medical images of the brain. However, not all techniques have been used for MRI form, which offers better resolution compared to other forms of radiological images of brain. Therefore, the prime aim of this solution is to offer a non-linear image enhancement process to make simple process of rectifying the artifacts irrespect of any form of images. The algorithm designed for this purpose is as shown below:

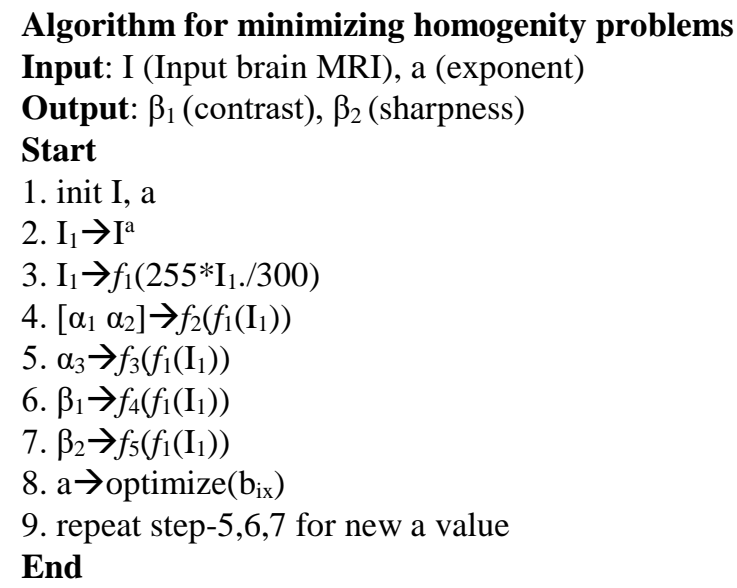

The homogenity problems in brain MRI is usually caused if the contrasts as well as sharpness are not uniformly maintained. The step-wise description of the proposed algorithm is as follows: The algorithm takes the input image (I) and exponential factor (a) as the input that after processing offers two significants outcome of $\beta_{1}$ (Input brain MRI) and $\beta_{2}$ (sharpness) (Line-1). The exponential variable $a$ is used for encoding and decoding operation in order to perform non-linearity operations (Line-2) to obtain a processed image $\mathrm{I}_{1}$. A set of simple discrete function $f_{1}(\mathrm{x})$ is designed for making it as unsigned integer of 8 bits (Line-3). This operation is followed by applying a specific function $f_{2}(\mathrm{x})$ in Line-4 in order to obtain a contrast-specific information from $I_{1}$. The algorithm also computes contrast per pixels $\alpha_{3}$ with an aid of function $f_{3}(\mathrm{x})$ in Line-5 with an input of gray scale image. It is obtained by calculating average of matrix elements for differential image that is extracted by applying convolution operation on grayscale image using kernel matrix (Line-5). Finally, two functions $\beta_{1}(\mathrm{x})$ and $\beta_{2}(\mathrm{x})$ are applied to obtain the contrast and sharpness in the processed image $\mathrm{I}_{1}$ (Line-6 and Line-7). The contrast is calculated as follows- it computes the mean of the input image than subtract the mean from main matrix of input image. The obtained difference is than squared followed by applying squareroot of summation of all the squared difference obtained in prior step. The contrast is obtained by dividing the square-root of sum to product of sizes of original images. The sharpness is obtained by applying approximated gradient to the double precision image. The square root of the summations of dual coefficients is obtained followed by dividing the sum of all the obtained square root value to number of matrix elements. This operation finally gives better sharpness to image. The next process is to perform optimization of both contrasts as well as sharpness factor. For this purpose, the exponential value a is further optimized using a new variable $b_{\mathrm{ix}}$ where $b_{\mathrm{ix}}$ represents a condition where summation of sharpness value is equal to maximum value of sharpness.

\subsection{Solution towards noise-related enhancement problem}

Denoising principles usually involves too many series of operation right from identification of noise types to preventing it. Hence, the proposed system contributes to offer a simple denoising principle that can perform removal of noise while balancing the image enhancement process simulataneously. Presence of significant noises e.g. multiplicative noise is quite inevitable in brain MRI images that also affect the brightness aspect of it. The present algorithm is capable of identifying the frequency domain of the illuminance as well as reflectance factor for addressing the enhancement and noise problem. The steps of the proposed algorithm are as follows: 


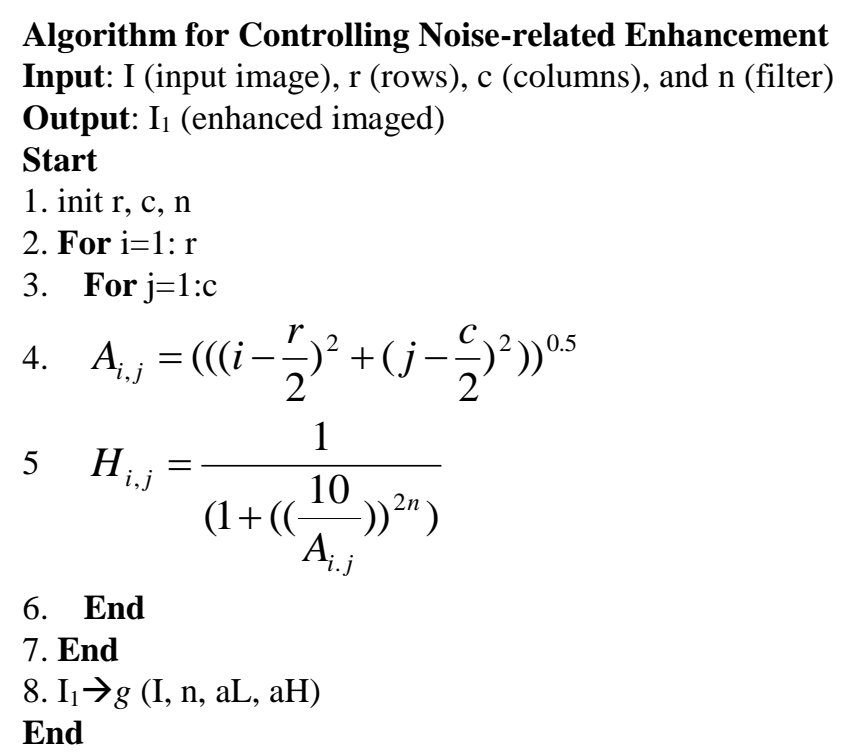

The algorithm takes the input image $I$ as well as $r$ (rows), $c$ (columns), and $n$ (filter). The proposed algorithm is used for enhancing the grayscale image of brain MRI with respect to its appearance. It does so by compression the illumination and improves the reflection aspect of the image. The algorithm considers computation for all the rows (Line-2) and all the columns (Line-3) to compute the empirical expression of the filter (Line-4 and Line-5). The algorithm also considers three user-defined inputs i.e. $\mathrm{n}$, aL, and aH in order to further executes a function $g$ (Line-8). The function $g(\mathrm{x})$ is responsible for optimizing the performance of the noise-removal operation in order to offer an output of enhanced image $I_{1}$ (Line-8). The enhancement process is as follows: -the input gray scaled image is changed to double precision image followed by extraction of size of this image to generate $r$ and $c$ values that is used in Line-2 and Line-3. The empirical expression in Line- 5 is executed to remove the artifacts with respect to the input arguments of $r$, $c$, and $n$ in order to generate filtered image $\mathrm{H}$.

$$
\mathrm{H} \rightarrow(\mathrm{aH}-\mathrm{aL}) * \mathrm{H})+\mathrm{aL}
$$

The next process is to obtain the logarithmic version of an image followed by performing Fast Fourier Transform operation on it. After applying inverse operation, the normalized image is obtained that is basically treated as the finally enhanced image of this operation.

\subsection{Solution towards simplistic in implementation strategy}

It was already noticed that existing approaches have used highly iterative operation while performing image classification, which is normally the last stage. The involvements of iterative steps are quite required as it is necessary to double check the final outcome by extracting the best outcome. However, this process of iteration can be minimized if necessary, care is taken in the pre-processing step itself. Therefore, the prime agenda of this algorithm is to offer a highly progressive as well as non-iterative approach for performing image enhancement. The steps of the algorithm are as follows:

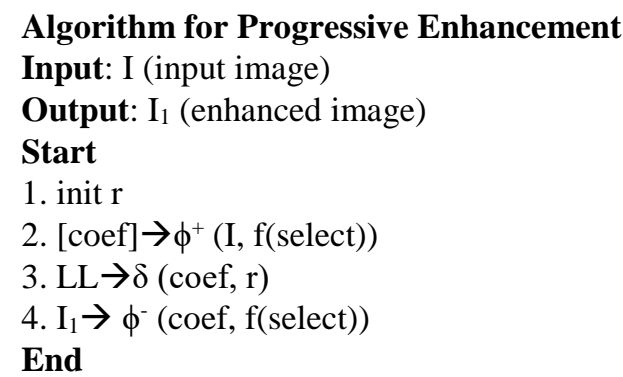

The algorithm takes the input of grayscaled brain MRI image that after processing gives the outcome of $\mathrm{I}_{1}$ (enhanced image). The algorithm also takes the $\mathrm{r}$ value as the input (Line-1) with a selection of user-defined filtering technqiues. A transformed-based technique is applied for the proposed system. 
A function two- dimensional function discrete wave transforms $\phi^{+}$is implemented considering the input argument of input image and a function to select the transformation technique (Line-2). The outcome for this process is generation of 4 different coefficients e.g. LL (Low-Low), LH (Low-high), HL (High Low), $\mathrm{HH}$ (High High). Hence, coef= (LL, LH, HL, HH). The next part of the algorithm is to offer further enhancement by targeting LL coefficient followed by applying inverse function on it. The function $\delta(\mathrm{x})$ is meant for this purpose (Line-3). Finally, for all the values of $\mathrm{r}$, the algorithm applies $\phi(\mathrm{x})$ as the optimized parameter to enhance it further. In order to perform optimization, following steps are conducted: perform normalization of image followed by grayscale thresholding to obtain highly enhanced image $\mathrm{I}_{1}$ (Line-4). Therefore, the algorithm is highly progressive and the enhancement offered is highly superior without inclusion of any computationally burdening task. The next section discusses the outcomes obtained.

\section{RESULTS ANALYSIS}

The analysis of the proposed system is carried out considering all the three algorithms i.e. Algorithm-1 (Algorithm for minimizing homogenity problems), Algorithm-2 (Algorithm for Controlling Noise-related Enhancement), and Algorithm-3 (Algorithm for Progressive Enhancement). The complete assessment was carried out both visually and graphically using standard brain MRI image dataset [34]

\subsection{Visual outcomes}

The visual outcomes Table 1 highlights that each algorithm offers different forms of visuality to the enhanced image. For similar set of given inputs, the outputs significant differs that seems to be highly specific to specific algorithms. However, each visual outcome is good enough for performing diagnostic and hence offer better flexibility in investgating the input of brain MRI. All the significant parameters e.g. contrast, brightness, and sharpness has been significantly improved for each outcomes of each algorithm.

Table 1. Visual outcomes of proposed system

\begin{tabular}{c} 
Algorithm-1 \\
Input Image \\
\hline$F$ Enhanced Image
\end{tabular}

\subsection{Graphical outcomes}

The assessment of numerical outcomes was carried out considering non-conventional enhancement processe.g. Global contrast factor, contrast per pixel, contrast, and sharpness. This parameter is considered as a direct representation of perception of an image by the human with respect to contrast. Using different levels of resolution, this parameter is used for calculating overall value of the contrast. The numerical outcome shows that algorithm-3 offers better Global Contrast factor in comparison to other two algorithms as shown in Figure 2. A closer look will show that algorithm-1 and algorithm-2 offers nearly similar performance. The actual inference to this graphical outcome will mean that the resultant image obtained by algorithm-3 is having perceptible contrast that can be practically sensed by any human, which is good from application viewpoint.

The next performance parameter considered for assessment is contrast per pixel that is calculated by mean difference of any point of a given pixel with its neighboring pixel refer Figure 3 . In this case, the 
outcome shows that contrast value offered by Algorithm-1 is more as compared to algorithm-2 and algorithm-3. However, the increased value of contrast will only mean human imperceptible image by exhibiting the image to be near to whithish. It will also mean that lowered value of contrast per pixel will mean a darker pixel, which is also human imperceptible. Hence, algorithm-1 is good for darker image while algorithm-2 is good for image with abnormally increased contrast ranges. However, algorithm-3 is found to offer a good balance between the higher and lower intensity and thereby it offers cumulatively good global contrast factor as seen in Figure 2.

Figure 4 highlights the comparative analysis of the all the three algorithms with respect to contrast with an agenda to see better capability to attain increased contrast value. The outcome shows that only algorithm-1 and algorithm-3 offers such capabilitytooffers contrast within better tolerablelimits of human perception. However, there is a discreet meanng for both of them as algorithm-1 is all about non-linear mechanism to control contrast while algorithm-3 includes various transformed-based steps followed by enhancing the pixels in LL region along with optimization. Hence, tendency to obtain better image is always good for algorithm-3 as compared to algorithm-1. On the other hand, algorithm-2 doesn't offer much flexibility in the calculation of $\mathrm{H}$ that results in less capability to perform increase in contrast.

Figure 5 shows the comparative analysis of all the images with respect to sharpness aspect. Although, sharpness is necessary for better image enhancement but higher degree of sharpness results in pixelated image in the outcome. This fact can be visible in graphical trend where algorithm-1 is found to show the highest sharpness while algorithm-3 is found to show slightly less sharp. Algorithm-3 cannot be considered to offer much higher sharpness.

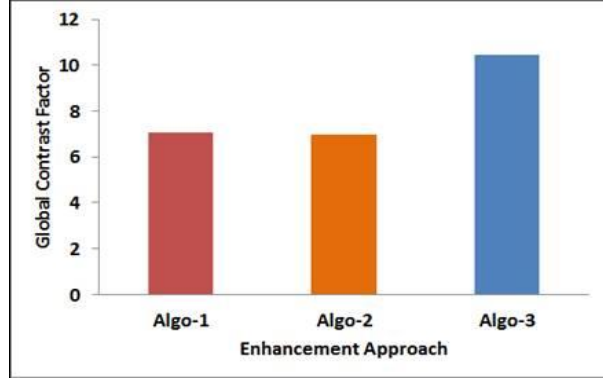

Figure 2. Comparative analysis of global contrast factor

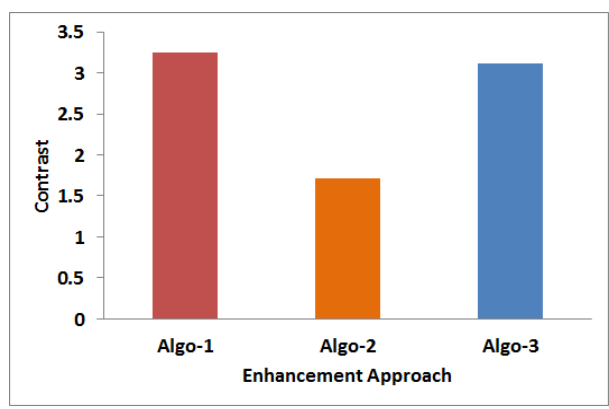

Figure 4. Comparative analysis of contrast

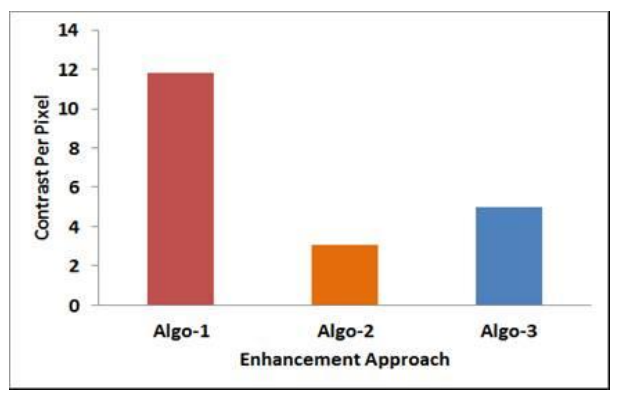

Figure 3. Comparative analysis of contrast per pixel

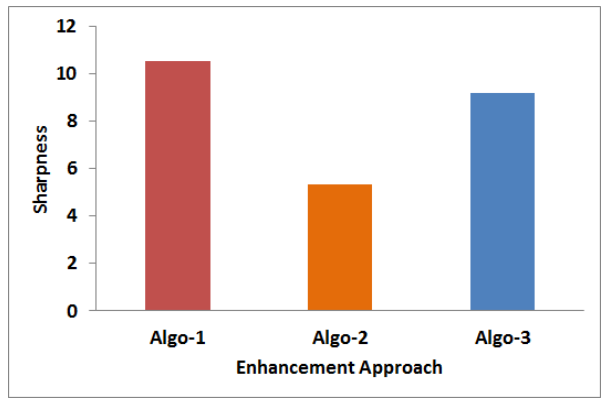

Figure 5. Comparative analysis of sharpness

\section{CONCLUSION}

The contributions of the proposed systems are: i) it offers three set of flexible image enhancement algorithm with optimized performance, ii) it is capable of balancing multiple aspects while performing image enhancement, iii) it is computationally faster for brain MRI images. It was found that for 10 MRI brain images, algorihm-1 took approximately $0.7665 \mathrm{sec}$, algorithm 2 took approximately $0.9283 \mathrm{sec}$, while algorithm-3 consumed approximately $0.4271 \mathrm{sec}$. Each algorithm has its own capability to perform image enhancement which completely depends upon the input image of brain MRI. Our future work will be in the direction of further continuting investigation on it to apply on disease identification problems. 


\section{REFERENCES}

[1] P. Vasuki, J. Kanimozhi and M. B. Devi, "A survey on image preprocessing techniques for diverse fields of medical imagery," 2017 IEEE International Conference on Electrical, Instrumentation and Communication Engineering (ICEICE), Karur, pp. 1-6, 2017.

[2] S. L. Jui et al., "Brain MRI Tumor Segmentation with 3D Intracranial Structure Deformation Features," in IEEE Intelligent Systems, vol. 31, no. 2, pp. 66-76, Mar.-Apr. 2016.

[3] Despotović, Ivana, Bart Goossens, and Wilfried Philips. "MRI segmentation of the human brain: challenges, methods, and applications," Computational and mathematical methods in medicine 2015 (2015).

[4] Kobashi, Syoji, László G. Nyúl, and Jayaram K. Udupa. "Soft Computing in Medical Image Processing," Computational and mathematical methods in medicine 2016, 2016.

[5] O. Ghribi, L. Sellami, M. Ben Slima, A. Ben Hamida, C. Mhiri and K. B. Mahfoudh, "An Advanced MRI MultiModalities Segmentation Methodology Dedicated to Multiple Sclerosis Lesions Exploration and Differentiation," in IEEE Transactions on NanoBioscience, vol. 16, no. 8, pp. 656-665, Dec. 2017.

[6] Hiralal, Reshma, and Hema P. Menon. "A Survey of Brain MRI Image Segmentation Methods and the Issues Involved," The International Symposium on Intelligent Systems Technologies and Application, Springer, Cham, 2016.

[7] L. Dora, S. Agrawal, R. Panda and A. Abraham, "State-of-the-Art Methods for Brain Tissue Segmentation: A Review," in IEEE Reviews in Biomedical Engineering, vol. 10, pp. 235-249, 2017.

[8] González-Villà, Sandra, et al. "A review on brain structures segmentation in magnetic resonance imaging," Artificial intelligence in medicine 73, 45-69, 2016.

[9] S. Poornachandra and C. Naveena, "Pre-processing of MR Images for Efficient Quantitative Image Analysis Using Deep Learning Techniques," 2017 International Conference on Recent Advances in Electronics and Communication Technology (ICRAECT), Bangalore, pp. 191-195, 2017.

[10] Roy, Sudipta, et al. "An improved brain MR image binarization method as a preprocessing for abnormality detection and features extraction," Frontiers of Computer Science 11.4 Springer, 717-727, 2017.

[11] A. Min and Z. M. Kyu, "MRI Images Enhancement and Tumor Segmentation for Brain," 2017 18th International Conference on Parallel and Distributed Computing, Applications and Technologies (PDCAT), Taipei, pp. 270-275, 2017.

[12] V. Alex, K. Vaidhya, S. Thirunavukkarasu, C. Kesavadas \& G. Krishnamurthi, "Semisupervised learning using denoising autoencoders for brain lesion detection and segmentation," Journal of Medical Imaging, Vol. 4(4), 041311, 2017.

[13] A. Ortiz, A. A. Palacio, J. M. Górriz, J. Ramírez, and D. S-González, "Research Article Segmentation of Brain MRI Using SOM-FCM-Based Method and 3D Statistical Descriptors," Hindawi Publishing Corporation Computational and Mathematical Methods in Medicine, pp. 12, 2013.

[14] S. Abbasi and F. Tajeri Pour, "A hybrid approach for detection of brain tumor in MRI images," 2014 21th Iranian Conference on Biomedical Engineering (ICBME), Tehran, 2014, pp. 269-274.

[15] S. Gupta and R. Porwal, "Research Article Appropriate Contrast Enhancement Measures for Brain and Breast Cancer Images," Hindawi Publishing Corporation International Journal of Biomedical Imaging, pp. 8, 2016

[16] B. H. Menze et al., "The Multimodal Brain Tumor Image Segmentation Benchmark (BRATS)," in IEEE Transactions on Medical Imaging, vol. 34, no. 10, pp. 1993-2024, Oct. 2015.

[17] N. B. Bahadure, A.K. Ray and H. P. Thethi, "Research Article Image Analysis for MRI Based Brain Tumor Detection and Feature Extraction Using Biologically Inspired BWT and SVM," Hindawi International Journal of Biomedical Imaging, pp. 12, 2017.

[18] P. Dhage, M. R. Phegade and S. K. Shah, "Watershed segmentation brain tumor detection," 2015 International Conference on Pervasive Computing (ICPC), Pune, pp. 1-5, 2015.

[19] A. Faisal, S. Parveen, S. Badsha and H. Sarwar, "An Improved Image Denoising and Segmentation Approach for Detecting Tumor from 2-D MRI Brain Images," 2012 International Conference on Advanced Computer Science Applications and Technologies (ACSAT), Kuala Lumpur, pp. 452-457, 2012.

[20] S. L. Jui et al., "Brain MRI Tumor Segmentation with 3D Intracranial Structure Deformation Features," in IEEE Intelligent Systems, vol. 31, no. 2, pp. 66-76, Mar.-Apr. 2016.

[21] H. Kaur and J. Rani, "MRI brain image enhancement using Histogram Equalization techniques," 2016 International Conference on Wireless Communications, Signal Processing and Networking (WiSPNET), Chennai, pp. 770-773, 2016.

[22] Y. Megersa and G. Alemu, "Brain tumor detection and segmentation using hybrid intelligent algorithms," AFRICON 2015, Addis Ababa, pp. 1-8, 2015.

[23] Parveen and A. Singh, "Detection of brain tumor in MRI images, using combination of fuzzy c-means and SVM," 2015 2nd International Conference on Signal Processing and Integrated Networks (SPIN), Noida, pp. 98-102, 2015.

[24] S. Pereira, A. Pinto, V. Alves and C. A. Silva, "Brain Tumor Segmentation Using Convolutional Neural Networks in MRI Images," in IEEE Transactions on Medical Imaging, vol. 35, no. 5, pp. 1240-1251, May 2016.

[25] C. H. Rao, P. V. Naganjaneyulu and K. S. Prasad, "Brain Tumor Detection and Segmentation Using Conditional Random Field," 2017 IEEE 7th International Advance Computing Conference (IACC), Hyderabad, pp. 807-810, 2017.

[26] N. Senthilkumaran and J. Thimmiaraja, "Histogram Equalization for Image Enhancement Using MRI Brain Images," 2014 World Congress on Computing and Communication Technologies, Trichirappalli, pp. 80-83, 2014. 
[27] Y. Sharma and Y. K. Meghrajani, "Brain tumor extraction from MRI image using mathematical morphological reconstruction," 2014 2nd International Conference on Emerging Technology Trends in Electronics, Communication and Networking, Surat, pp. 1-4, 2014.

[28] S. N. Sulaiman, S. M. C. Ishak, I. S. Isa and N. Hamzah, "De-noising of noisy MRI brain image using the switchingbased clustering algorithm," 2014 IEEE International Conference on Control System, Computing and Engineering (ICCSCE 2014), Batu Ferringhi, pp. 1-6, 2014.

[29] K. Xiao, A. E. Hassanien, Y. Sun and E. K. K. Ng, "Brain MR image tumor segmentation with ventricular deformation," 2011 IEEE 7th International Conference on Intelligent Computer Communication and Processing, Cluj-Napoca, 2011, pp. 213-217.

[30] V. Zeljkovic et al., "Automatic brain tumor detection and segmentation in MR images," 2014 Pan American Health Care Exchanges (PAHCE), Brasilia, pp. 1-1, 2014.

[31] Hamiane, Madina, and Fatema Saeed. "SVM Classification of MRI Brain Images for Computer-Assisted Diagnosis." International Journal of Electrical and Computer Engineering (IJECE) 7, no. 5, 2555-2564, 2017.

[32] Jung, Se-Hoon, Jong-Chan Kim, and Chun-Bo Sim. "Prediction data processing scheme using an artificial neural network and data clustering for Big Data." International Journal of Electrical and Computer Engineering (IJECE), vol. 6, no. 1, 330-336, 2016.

[33] Hussein Attya Lafta, Zainab Falah Hasan, Noor Kadhim Ayoob, "The classification of medical datasets using back propagation neural network powered by genetic-based features elector," International Journal of Electrical and Computer Engineering (IJECE), vol. 9, no. 2, 2017.

[34] Gorgolewski, K.J., Wheeler, K., et al., "The impact of shared data in neuroimaging: the case of OpenfMRI.org." 2015. Available at: http://dx.doi.org/10.7490/f1000research.1110040.1.

\section{BIOGRAPHIES OF AUTHORS}

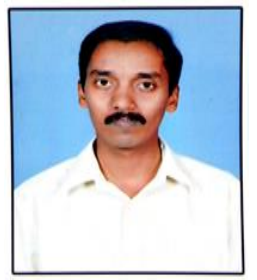

Harish S., I have completed B.E. in Telecommunication Engineering from Bangalore University, and M. Tech in Information \& Communication Systems from Visvesvaraya Technological University. Pursuing Ph. D in Electrical Engineering from Visvesvaraya Technological University. I am having 13 years of teaching experience. My area of research is Bio-medical Image Processing.

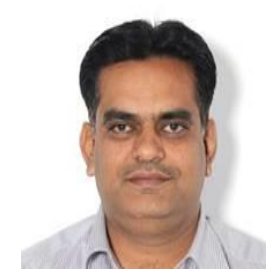

G. F. Ali Ahammed working as an Associate Professor \& HOD in the Department of Digital Electronics \&Communication systems at VTU PG centre, Mysuru. He is having 15 years of Teaching and Research Experience. He pursued Ph.D., Computer Science \& Technology, from Sri Krishna Devaraya University, Anantapur, Andra Pradesh. Master of Technology, Networking \& Internet Engineering, from Visvesvaraya Technological University, Belagavi, Bachelor of Engineering in Electronics \& Communication Engineering, from Bangalore University, Bangalore. He worked as BOE member for Visvesvaraya Technological University, Belagavi. He is also BOE member for SJCE Mysore \& NIE Mysore, Autonomous Colleges. He is BOS member for NIE Autonomous college. He is Local Inspection Committee (LIC) member for VTU, Belagavi. He has published several Research papers in International, National Journals/conferences. He is a Member of CSI, IAENG, IBM Academic Initiative, and LM ISTE. Dr. G.F. Ali Ahammed area of interest and research include Networking, Performance enhancement algorithms, Cloud Computing, cryptography and Communication. He has the Recipient of Seed money Young Scientist Research Award (SMYSR) from Vision Group of Science and Technology (VGST), Bangalore. He is also fetched various Grants from VGST\&AICTE. 